\section{ORIGINAL RESEARCH}

K. Aydin

A. Ucar

K.K. Oguz

O.O. Okur

A. Agayev

Z. Unal

S. Yilmaz

C. Ozturk

\title{
Increased Gray Matter Density in the Parietal Cortex of Mathematicians: A Voxel-Based Morphometry Study
}

\begin{abstract}
BACKGROUND AND PURPOSE: The training to acquire or practicing to perform a skill, which may lead to structural changes in the brain, is called experience-dependent structural plasticity. The main purpose of this cross-sectional study was to investigate the presence of experience-dependent structural plasticity in mathematicians' brains, which may develop after long-term practice of mathematic thinking.
\end{abstract}

\begin{abstract}
MATERIALS AND METHODS: Twenty-six volunteer mathematicians, who have been working as academicians, were enrolled in the study. We applied an optimized method of voxel-based morphometry in the mathematicians and the age- and sex-matched control subjects. We assessed the gray and white matter density differences in mathematicians and the control subjects. Moreover, the correlation between the cortical density and the time spent as an academician was investigated.
\end{abstract}

\begin{abstract}
RESULTS: We found that cortical gray matter density in the left inferior frontal and bilateral inferior parietal lobules of the mathematicians were significantly increased compared with the control subjects. Furthermore, increase in gray matter density in the right inferior parietal lobule of the mathematicians was strongly correlated with the time spent as an academician $(r=0.84 ; P<.01)$. Left-inferior frontal and bilateral parietal regions are involved in arithmetic processing. Inferior parietal regions are also involved in high-level mathematic thinking, which requires visuospatial imagery, such as mental creation and manipulation of 3D objects.
\end{abstract}

CONCLUSION: The voxel-based morphometric analysis of mathematicians' brains revealed increased gray matter density in the cortical regions related to mathematic thinking. The correlation between cortical density increase and the time spent as an academician suggests experience-dependent structural plasticity in mathematicians' brains.

G enetic, epigenetic, and environmental factors interact to produce the fine structure of the human brain. ${ }^{1-4}$ Genetically determined cortical architecture may need to undergo functional and structural changes to meet the increasing demands of a complex environment. The training to acquire or practicing to perform a skill may lead to structural changes in the brain, which is called experience-dependent structural plasticity. A previous MR imaging study revealed that memorizing a huge amount of spatial information increased the cortical gray matter density in the right hippocampus of the human brain, which represents the spatial navigation center. ${ }^{1}$ Thus, the human brain can make regional structural reorganizations associated with experience and learning., ${ }^{3,5-8}$

Music is one of the most fascinating products of the human brain. Because becoming a professional musician requires a long, intense, and highly specific training during the early periods of brain maturation, the musician's brain has been used as a model to study the development of experience-dependent structural plasticity. ${ }^{9,10}$ During their professional training period, musicians learn to convert musical symbols to complex motor actions, memorize long and complex musical artworks,

Received January 25, 2007; accepted after revision April 11.

From the Departments of Neuroradiology (K.A.) and Radiology (A.U., A.A., S.Y.) Istanbul Medical School, Istanbul University, Capa, Istanbul, Turkey; Department of Radiology (K.K.O.), Hacettepe University, Sihhiye, Ankara, Turkey; Institute of Biomedical Engineering (0.0.0., C.0.), Bogazici University, Bebek, Istanbul, Turkey and Department of Radiology (Z.U.), KVK American Hospital, Nisantasi, Istanbul, Turkey.

Please address correspondence to Kubilay Aydin, Adnan Saygun caddesi, M. Salih Rustubey sokak, Ulus Konaklari, No:8/12, Ulus, İstanbul, Turkey; e-mail: dr.aydink@superonline.com or aydink@istanbul.edu.tr

DOI 10.3174/ajnr.A0696 and acquire the capability of perfect tone discrimination. The performances of these very complex tasks cause experiencedependent adaptive changes in the task-related regions of their brain. ${ }^{9,11-16}$ The morphometric MR studies conducted in the musicians revealed the presence of increased gray matter density in the motor and auditory cortex and the cerebellum compared with nonmusicians. ${ }^{15}$ It has been proposed that longterm and intense practice by pianists to perform sequential motor tasks with high accuracy resulted in experience-dependent adaptive changes in the related regions of their brains. Results of another voxel-based morphometric study revealed increased gray matter density in the left inferior frontal gyrus (Broca area) of orchestra musicians. ${ }^{17}$ The authors of the latter study hypothesized that long-term professional activity to increase visuospatial and audiospatial abilities led to increased gray matter density in the Broca area of orchestra musicians.

Doing mathematics is another unique feature of the brain. Mathematicians have to go through very intense and focused training. After their formal graduate education, mathematicians continue to spend hours, days, and even weeks to concentrate on solving mathematic problems or to create a mathematic model to explain the social and economic events throughout their professional life. In this study, we aimed to investigate whether the long-term intense training and practicing of mathematic thinking could induce structural plasticity in a mathematician's brain. We hypothesized that highly concentrated, focused, and professionally practiced mathematic thinking by mathematicians may create an enriched environment effect to develop experience-dependent structural plastic changes in their brains. To test our hypothesis, we 


\begin{tabular}{lcc}
\hline $\begin{array}{l}\text { Table 1: Demographic data of the mathematicians and control } \\
\text { subjects }\end{array}$ \\
\hline Data & Mathematicians & $\begin{array}{c}\text { Control } \\
\text { Subjects }\end{array}$ \\
\hline Age, y & $35.50 \pm 6.95$ & $35.43 \pm 7.62$ \\
Sex (female/male) & $9 / 17$ & $7 / 16$ \\
Period of time spent as an academic, y & $13.65 \pm 6.95$ & $14.13 \pm 7.16$ \\
\hline
\end{tabular}

investigated the possible gray and white matter density differences between a group of professional mathematicians (academicians at mathematics departments of universities) and the control subjects.

\section{Materials and Methods}

\section{Subjects}

We studied 26 mathematicians (19 men and 7 women; mean age, $35.50 \pm 6.80$ years) who were recruited from the volunteers who have been working as academicians at departments of mathematics. All of the mathematicians completed a 4 -year undergraduate program of mathematics and had passed a 4-year-long PhD education program. Four of them were involved in algebra, 14 in applied mathematics, 3 in probabilistic analysis, and 2 in mathematic physics. The native language of all of the mathematicians and control subjects was Turkish. All of the mathematicians and control subjects could speak only English as a foreign language. The mathematicians and control subjects were neither interested in music at a professional level nor could play a musical instrument. One mathematician who was more than 60 years of age and another who could play piano were not included in the study. Exclusion criteria for the mathematicians and control subjects also included any contraindication for MR imaging; alcohol or drug abuse; and any history of neurodegenerative disease, seizure, central nervous system infection, cerebrovascular disease, diabetes mellitus, and head trauma causing loss of consciousness that lasted more than 30 minutes or that required hospitalization. Cerebral lesions other than nonspecific cerebral white matter spots, present on T2-weighted images, were also accepted as exclusion criteria. The mean period of time spent as an academician after completing bachelor's degree was $13.65 \pm 6.95$ years. The demographic data of the participants are summarized in Table 1.

We recruited 23 sex- and age-matched control subjects (16 men and 7 women) among the volunteers of the academicians from the faculties of medicine and philosophy (mean age, $35.43 \pm 7.62$ years). All of the mathematicians and control subjects were right-handed (Edinburgh handedness inventory). Informed consent was taken from all of the participants, and the study was approved by the local human subject committee.

\section{Image Acquisition}

Cranial MR imaging studies were performed on a $1.5 \mathrm{~T}$ superconducting whole-body MR imaging system (Symphony Maestro; Siemens Medical Systems, Erlangen, Germany) with a standard quadrature head coil. High-resolution anatomic images of the whole brain were acquired from the mathematicians and control subjects with T1weighted magnetization-prepared rapid acquisition gradient echo sequence $(\mathrm{TR}=11.08 \mathrm{~ms} ; \mathrm{TE}=4 \mathrm{~ms} ; \mathrm{TI}=300 \mathrm{~ms}$; relaxation delay time $=500 \mathrm{~ms}$; flip angle $=15^{\circ} ; \mathrm{FOV}=256 \times 192 \mathrm{~mm}$; matrix size $=$ $256 \times 192$ ) yielding 128 sagittal sections with a defined voxel size of $1 \times 1 \times 1.3 \mathrm{~mm}$. We also obtained the axial fast spin-echo T2weighted images (TR $=9200 \mathrm{~ms}$; TE $=110 \mathrm{~ms}$; the number of acqui- sitions $=3$ ) from all of the subjects to search for any pathologic findings defined in the study exclusion criteria.

\section{Voxel-Based Morphometry Protocol and Data Preprocessing}

The data preprocessing and analyses were performed with SPM2 (http://www.fil.ion.ucl.ac.uk/spm/software/spm2; Wellcome Department of Cognitive Neurology, London, United Kingdom) running under Matlab (MathWorks, Natick, Mass). MR scanning produces images in Digital Imaging and Communications in Medicine (DICOM) format. All of the images in DICOM format were converted to ANALYSE format with the software MRIcro ${ }^{18}$ (www.mricro. com). We applied an optimized method of voxel-based morphometry. Preprocessing of the data involved spatial normalization of all of the images into a standardized anatomic space, segmentation into gray and white matter, modulation, and spatial smoothing with a Gaussian kernel. ${ }^{19-21}$ To reduce the scanner-specific bias, we created a customized gray matter by averaging the smoothened and normalized images of all of the participants. T1-weighted MR images were normalized to the standard T1 template of the Montreal Neurologic Institute (MNI). The normalized images were smoothed and averaged to obtain a study-specific $\mathrm{T} 1$ template. All of the original structural MR images in native space were then normalized to this study-specific template. The normalized images were segmented into CSF, gray matter, and white matter compartments using the SPM2 priors. Afterward, CSF, gray matter, and white matter images were smoothened with an 8-mm full width at half maximum (FWHM) kernel and averaged to obtain study-specific CSF, gray matter, and white matter priors for later segmentation of native images. The original T1weighted images were segmented with the study-specific $\mathrm{T} 1$ template and gray matter, white matter, and CSF priors. This segmentation step involves an affine transformation of each scan to the template with a subsequent back projection into native space. We also performed a correction for volume changes (modulation) by modulating each voxel by the Jacobian determinants derived from the spatial normalization. An automated brain extraction procedure that incorporated a segmentation step was used to remove nonbrain tissue. The extracted gray matter images were then normalized to the groupspecific gray matter template. The normalization parameters were then applied to the original structural images in native space to reduce any contribution from nonbrain voxels and afford optimal spatial normalization of gray matter. These normalized images were segmented into gray and white matter. Finally, the normalized and segmented images were smoothed with a 10-mm FWHM isotropic Gaussian kernel.

\section{Statistical Analysis}

We investigated the regionally specific gray and white matter density differences between the mathematician and control subjects using voxel-by-voxel analysis of variance test. Because the groups did not show a significant difference in age and sex distribution, we did not enter these variables as covariates. For the statistical analysis, we excluded all of the voxels with a gray or white matter value below 0.2 ( of a maximum value of 1) to avoid possible edge effects around the border between gray and white matter and to include only voxels with sufficient gray and white matter, respectively. The findings were considered significant at a voxel level of $P<.0001$, uncorrected for multiple comparisons, with an extended threshold looking for clusters with at least 100 contiguous voxels. The relationship between the time duration of professional work and cortical gray matter density 


\begin{tabular}{|c|c|c|c|c|}
\hline \multicolumn{5}{|c|}{$\begin{array}{l}\text { Table 2: Regions with increased grey matter density in the } \\
\text { mathematicians }\end{array}$} \\
\hline \multirow{2}{*}{$\begin{array}{l}\text { Anatomic Region (Brodmann } \\
\text { Area) }\end{array}$} & \multicolumn{3}{|c|}{$\begin{array}{c}\text { Mathematicians } \\
\text { vs Controls } \\
\text { Talaraich } \\
\text { Coordinates } \\
\end{array}$} & \multirow{2}{*}{$\begin{array}{l}\text { Maximum } z \\
\text { Values }\end{array}$} \\
\hline & $\mathrm{x}$ & $y$ & $\bar{z}$ & \\
\hline Right inferior parietal lobule (BA 4) & 54 & -58 & 38 & 4.18 \\
\hline Right inferior parietal lobule (BA 7) & 32 & -46 & 60 & 4.08 \\
\hline Right inferior parietal lobule (BA 39) & 57 & -60 & 34 & 4.00 \\
\hline Left inferior parietal lobule (BA 40) & -38 & -50 & 57 & 3.74 \\
\hline Left inferior frontal gyrus (BA 46) & -42 & -34 & 12 & 3.51 \\
\hline
\end{tabular}

changes was investigated by regression analysis $(P<.05$, family-wise error [FWE] corrected). For localizing purposes, the MNI coordinates were converted to Talaraich coordinates using a dedicated script (mni2tal.m; available at http://www.mrc-cbu.cam.ac.uk/Imaging/ Common/mnispace.shtml). Wherever a significant correlation was found between the mathematicians and control subjects, the gray matter density values were extracted for each of these voxel locations into SPSS for Microsoft Windows (SPSS, Chicago, Ill), and a linear regression analysis was performed to calculate the correlation coefficient. The statistical analyses in this study were carried out by a statistician.

\section{Results}

No significant difference was observed in education length and the parental education levels between mathematicians and control subjects (Table 1). In comparison with the ageand sex-matched control subjects, the mathematicians had significantly higher gray matter densities in the right inferior parietal lobule (Talaraich coordinates: $\mathrm{x}=54, \mathrm{y}=-58, \mathrm{z}=38$ [Brodmann area, BA 4]; $\mathrm{x}=32, \mathrm{y}=-46, \mathrm{z}=60$ [BA 7]; $\mathrm{x}=$ $57, y=-60, z=34[B A 39])$; left inferior parietal lobule $(x=$ $-38, y=-50, z=57$ [BA 40]); and the left inferior frontal gyrus ( $\mathrm{x}=-42, \mathrm{y}=-34, \mathrm{z}=12$ [BA 46]; Table 2; Fig 1). There was no region of decreased gray matter density in the mathematicians' brains compared with control subjects. The regression analyses revealed that the period of time spent as an academician was significantly associated with the gray matter density in the right parietal lobules of the mathematicians (Fig $2 A)$. The gray matter density values extracted from the right inferior parietal lobule $(\mathrm{x}=57, \mathrm{y}=-60, \mathrm{z}=34$ [BA 39]; $z=$ 7.28; $P<.05$; FEW corrected) showed a strong correlation with the period of time spent as an academician $(r=0.84 ; P<$ .01 ) on the SSPS correlation analysis (Fig $2 B$ ). The analysis of images revealed no difference in the white matter density of mathematicians and the control subjects.

\section{Discussion}

The relationship between the morphology and functions of the human brain has attracted the interest of neuroscientists since the beginning of 20th century. ${ }^{22,23}$ Postmortem studies of some famous musicians, intellectual political leaders, and scientists were reported in search of the associations between the outstanding skills and the extraordinary cerebral morphology. ${ }^{24-26}$ In some of these reports, the famous musicians had larger supramarginal gyrus than the normal population. ${ }^{25}$ The postmortem study of Albert Einstein's brain showed that his inferior parietal lobule was larger than that of the control subjects and had a very exceptional parietal gyral morphology. ${ }^{26}$ It has been speculated that there might be an association between the exceptionally enlarged inferior parietal lobules and his outstanding creativity in mathematic thinking. Similarly, the postmortem study of the brain of another famous mathematician, Johann Karl Friedrich Gauss, revealed a remarkably convoluted appearance of the parietal lobes. ${ }^{22,24} \mathrm{To}-$ day, we know from the recent in vivo morphometric MR studies that the morphology of the human brain is determined by genetic factors but may also be modified by experiences.

Several cross-sectional morphometry studies have been conducted in musicians to investigate the morphologic differences of their brains from normal control subjects and to search the association between morphologic differences and the training and practicing of musical skills. ${ }^{9-14,16}$ Results of these studies revealed that musicians had anatomic differences in several regions of their brain that have been involved in motor and auditory processing. The morphologic differences observed in musicians' brains were significantly correlated with the musical skills and ages of commencement for musical training. ${ }^{13,14}$ Amunts et $\mathrm{al}^{13}$ reported a strong negative correlation between the commencement age of musical training and sizes of hand-motor cortical regions of pianists. In another study, musicians who began the musical training earlier than age 7 years had larger anterior corpus callosums than the musicians who started the training later. ${ }^{11}$ Musicians with an absolute pitch skill (ability to discriminate musical tones without a need for a reference tone) had larger planum temporale than the musicians without an absolute pitch. ${ }^{14}$ Because they are strongly associated with training periods and professional skills, it has been proposed that morphologic differences observed in the brains of musicians were experience-dependent structural changes developed as a result of intense, long-term training and practicing musical skills. ${ }^{9}$ In the study conducted with the taxi drivers in London, Maguire et $\mathrm{al}^{1}$ reported a significant association between the increased gray matter density in the right posterior hippocampus of the taxi drivers and the period of time spent as a taxi driver. They proposed that long-term training and practicing to memorize a huge amount of spatial information might cause an experience-dependent structural change in the hippocampus of the taxi drivers in which the spatial information is processed. They also found that the control subjects had larger gray matter volumes in the anterior hippocampus. They hypothesized that extensive navigation experience led to redistribution of gray matter in the hippocampus. In our study, we did not observe any finding that might indicate the presence of gray matter redistribution in the mathematicians. Recent longitudinal morphometry studies demonstrated that repeated practicing of a visuomotor task and intense learning of abstract information could lead to gray matter density changes in task-related regions of brain. ${ }^{3,7,27}$ In summary, the results of cross-sectional and longitudinal in vivo morphometric MR imaging studies point to the presence of experience-dependent structural plasticity in the human brain.

Voxel-based morphometry is a statistical imaging method that gives opportunities to investigate regionally specific subtle gray matter differences between various study groups. ${ }^{19}$ To the best of our knowledge, this is the first in vivo study investigating morphologic differences of mathematicians' brains. 


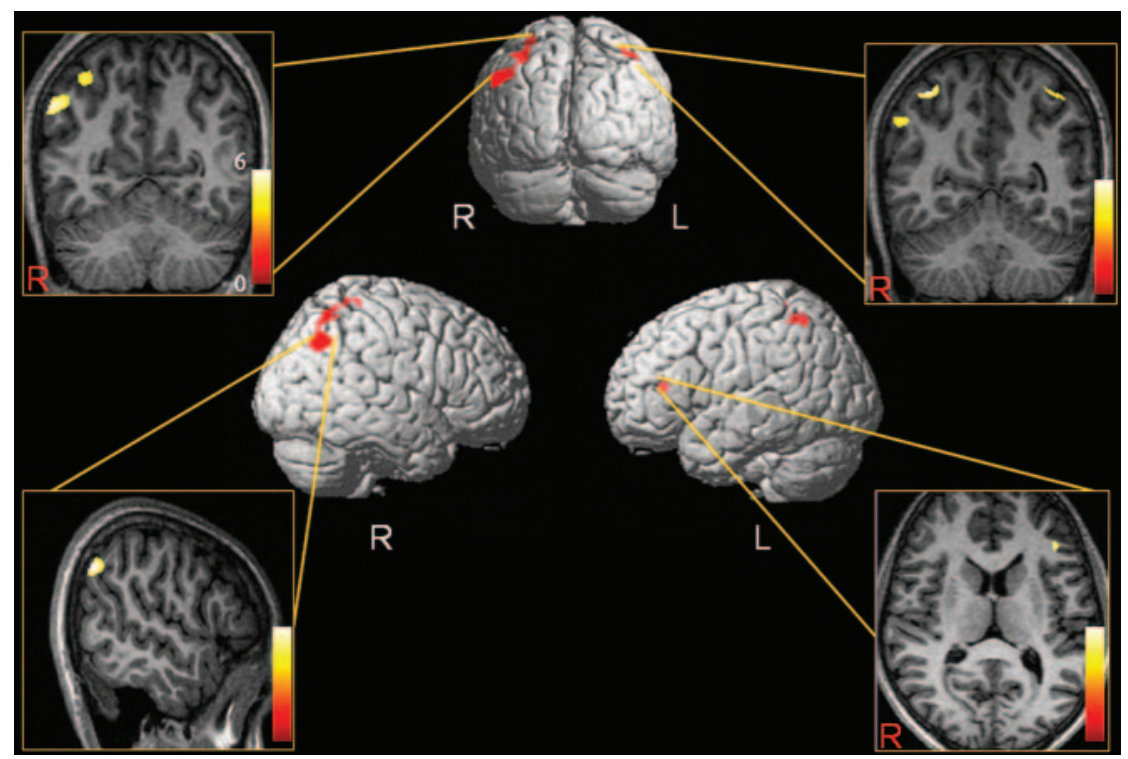

Fig 1. Distribution of significant voxels with increased gray matter density in the mathematicians relative to the control subjects (statistical significance is thresholded at $P<.0001$, uncorrected). Only clusters of voxels consisting of at least 100 voxels are displayed. The 3D overlay images demonstrate the regions with significantly increased gray matter density (red-labeled regions) in the mathematicians compared with the control subjects. The anatomic sectional images show the overlay of the results on the normalized T1-weighted images of a mathematician. The color scale on the T1-weighted images shows the ranges of $T$ values.
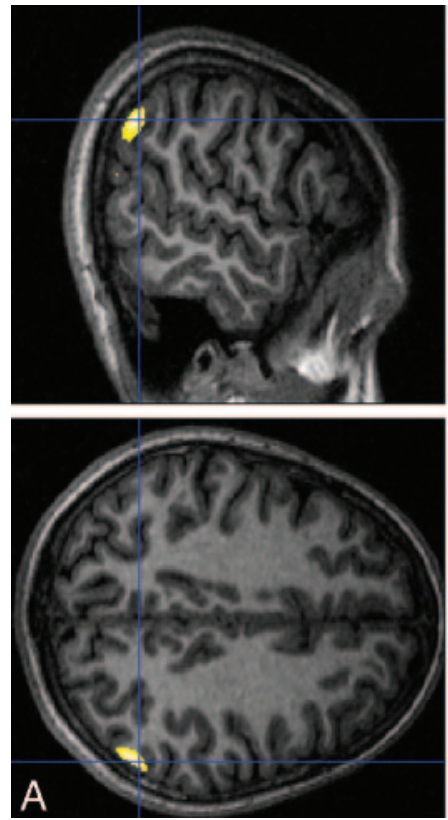
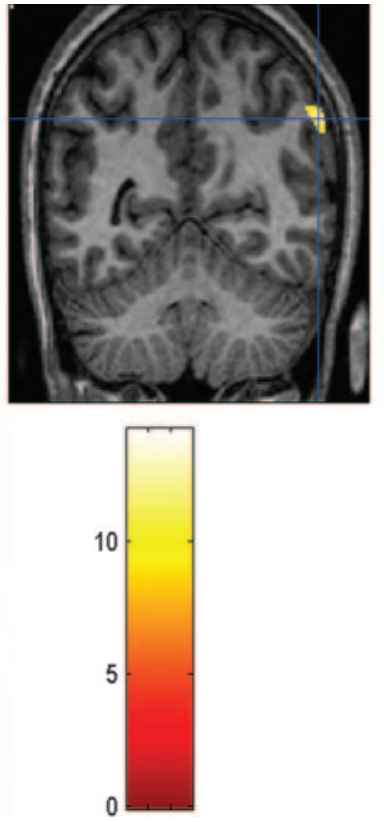

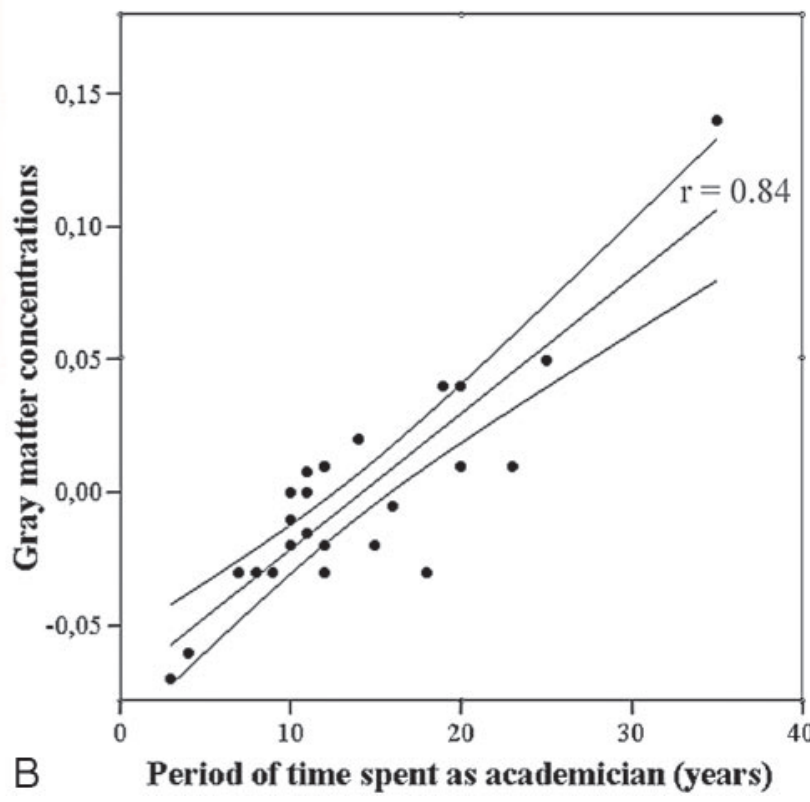

B Period of time spent as academician (years)

Fig 2. $A$, The result of the regression analysis testing the correlation between gray matter density of the mathematicians and period of time spent as an academician is overlayed on the normalized T1-weighted images. They show that gray matter density in the right inferior parietal region (Talaraich coordinates: $x=57, y=-60, z=34$ ) of the mathematicians is strongly correlated with the duration of time spent as an academician $(z=7.28 ; P<.05$, FWE corrected). $B$, The gray matter density values from the voxels showing the maximum correlation on the SPM regression analysis are extracted into SPSS. The scatter-plot graph shows the linear regression between the gray matter density and the duration of time spent as an academician $(r=0.84 ; P<.01)$. The middle line represents the linear regression, and the curves around it represent the $95 \%$ confidence intervals.

We hypothesized that long-term and specific training of mathematics and practicing of mathematic thinking might cause an experience-dependent structural alteration in mathematicians' brains. We conducted an optimized voxel-based morphometry study to test our hypothesis. ${ }^{20,21}$ Our results suggest that gray matter density of mathematicians shows diverse distribution in the inferior parietal lobules and left inferior frontal gyrus compared with age- and sex-matched control subjects. In addition, we found a strong correlation between the gray matter density in the right inferior parietal lobule of the mathematicians and the period of time spent as an academician. We know from previous functional MR imaging studies that processing of mental arithmetic calculations has language- and visuospatial-related components, which take place in different regions of the brain. ${ }^{28-30}$ The exact arithmetic calculation skills, such as multiplying, have languagespecific representations in the brain and are processed in the language-related left inferior frontal and left angular gyri. The arithmetic calculations requiring approximations and comparison of numbers, such as subtraction, have visuospatial representations in the brain and are processed in the bilateral parietal regions around the intraparietal sulci. In our study, we found significant gray matter density increase in the same regions where the mental arithmetic calculations are processed. The strong correlation between gray matter density change in the right inferior parietal lobule and the period of time spent as an academician indicates that structural difference observed in this region may result from an experience-dependent process. 
Long-term and intense mental practice with numeric data may lead to experience-dependent plastic changes in the related regions of brain. Mathematicians not only do arithmetic calculations, but they also deal with advanced mathematic problems that require mental imagery, mental creativity, and manipulation of 3D objects, which are all processed in parietal lobes. Mathematicians have a unique ways of scientific thinking, which they use in problem solving. It was stated in a selfdescription by Albert Einstein as, “... Words and language whether written or spoken, do not seem to play any part in my thought process. The psychological entities that serve as building blocks from my thought are certain signs or images, more or less clear, that I can reproduce or recombine at will." ${ }^{31}$ In his statements, he emphasized the visuospatial rather than verbal type of scientific thinking. Another famous mathematician, Jacques Hadamard, declared that his scientific thinking mainly relied on visuospatial rather than verbal processes. Throughout their academic lives, mathematicians practice this visuospatial data processing, which may contribute to the increased gray matter density in the parietal lobules. ${ }^{26}$

The gray matter changes observed in the left inferior parietal lobule and left inferior frontal gyrus did not correlate with the period of time spent as a mathematician. However, this does not rule out the possibility that gray matter density differences, which are observed in the left inferior frontal gyrus and left inferior parietal lobule, are experience-dependent structural changes. Results of recently reported longitudinal studies revealed that experience-dependent structural changes may develop within weeks or months after the stimuli. ${ }^{7,27}$ Structural changes observed in left inferior frontal gyrus and left inferior parietal lobule may develop in earlier times of the professional careers of mathematicians. Left inferior frontal gyrus is a language-related region of arithmetic processing. It would be good to test performances on verbal-related arithmetic calculations and to investigate the association between the gray matter density and test performances. Because most of the participants were not willing receive such a test, we were not able to investigate this association.

Histologic correlation of experience-dependent adaptive plasticity in the human brain has not been exactly defined yet. Neurogenesis, microglia proliferation, synaptogenesis, angiogenesis, axonal sprouting, modulation of synaptic transmission, and change in excitatory/inhibitory transmission balance have been postulated as the possible mechanisms of experience-dependent plasticity. ${ }^{32-45}$ Adaptive neurogenesis in the adult brain was first reported in animal studies. Food hiding and seeking behavior in food-storing bird species, which requires the storage of spatial information, stimulates the neurogenesis in the hippocampal region known as the spatial navigation center. ${ }^{38,44}$ Male canaries sing during their breeding season and learn new songs every year. Just before their breeding season, the high vocal center (a region in the brains of birds that is related to singing) of male canaries enlarges with the addition of new neurons. ${ }^{38,45}$ It has also been demonstrated that enriched environments in the experimental conditions may stimulate the neurogenesis in the hippocampus of adult mammalian brains. ${ }^{43}$ Synaptogenesis is the other mechanism that has been postulated to explain the mechanism of experience-dependent plasticity. ${ }^{32,42,43,46}$ Long-term sensory stimuli increase the number of synapses that modify the sensitivity of sensory system. Thus, newly generated synapses increase the complexity of the neuronal network that may enable the processing of complex data.

It is known from the previous studies that intelligence quotient (IQ) levels of individuals may influence the cortical gray matter distribution. It would be good to perform IQ measurements and consider IQ scores as a cofactor in the statistical analyses (analysis of covariance) of voxel-based morphometry. However, most of our subjects did not agree to get an IQ test. However, there was no significant difference in the education levels between the study groups, and all of the subjects in both groups were academicians. So, the individuals from both groups would be expected to have similar IQ levels. There is no objective evidence for a significant IQ level difference between the mathematicians and control subjects.

\section{Conclusion}

In conclusion, we found that mathematicians had significantly increased cortical gray matter density in the bilateral inferior parietal lobules and left inferior frontal gyrus, which are known to be involved in arithmetic calculations and visuospatial processing. Significant association of gray matter density changes in the right inferior parietal lobule with the period of time spent as an academician proposed that the structural change in this location is an experience-dependent process, which might be caused by the long-term practices of visuospatial and arithmetic data processing.

\section{References}

1. Maguire EA, Gadian DG, Johnsrude IS, et al. Navigation-related structural change in the hippocampi of taxi drivers. Proc Natl Acad Sci USA 2000;97:4398-403

2. Thompson PM, Cannon TD, Narrm KL, et al. Genetic influences on brain structure. Nat Neurosci 2001;4:1253-58

3. Draganski B, Gaser C, Busch V, et al. Neuroplasticity: changes in grey matter induced by training. Nature 2004;427:311-12

4. Hulshoff Pol HE, Schnack HG, Posthuma D, et al. Genetic contributions to human brain morphology and intelligence. J Neurosci 2006;26:10235-42

5. Terrazas A, McNaughton BL. Brain growth and the cognitive map. Proc Natl Acad Sci U S A 2000;97:4414-16

6. Mechelli A, Crinion JT, Noppeney U. Neurolinguistics: structural plasticity in the bilingual brain. Nature 2004;431:757

7. Draganski B, Gaser C, Kempermann G. Temporal and spatial dynamics of brain structure changes during extensive learning. J Neurosci 2006;26:6314-17

8. May A, Gaser C. Magnetic resonance-based morphometry: a window into structural plasticity of the brain. Curr Opin Neurol 2006;19:407-11

9. Munte TF, Altenmuller E, Jancke L. The musician's brain as a model of neuroplasticity. Nat Rev Neurosci 2002;3:473-78

10. Schlaug G. The brain of musicians. A model for functional and structura adaptation. Ann N Y Acad Sci 2001;930:281-99

11. Schlaug G, Jancke $L$, Huang $Y$, et al. In vivo evidence of structural brain asymmetry in musicians. Science 1995;267:699-701

12. Schlaug G, Jancke L, Huang Y, et al. Increased corpus callosum size in musicians. Neuropsychologia 1995;33:1047-55

13. Amunts K, Schlaug G, Jancke L, et al. Motor cortex and hand motor skills: structural compliance in the human brain. Hum Brain Mapp 1997;5:206-15

14. Keenan JP, Thangaraj V, Halpern AR, et al. Absolute pitch and planum temporale. NeuroImage 2001;1402-08

15. Gaser C, Schlaug G. Brain structures differ between musicians and non-musicians. J Neurosci 2003;23:9240-45

16. Hutchinson S, Lee LH, Gaab N, et al. Cerebellar volume of musicians. Cereb Cortex 2003;13:943-49

17. Sluming V, Barrick T, Howard M, et al. Voxel-based morphometry reveals increased gray matter density in Broca's area in male symphony orchestra musicians. NeuroImage 2002;17:1613-22

18. Rorden C, Brett M. Stereotaxic display of brain lesions. Behav Neurol 2000;12:191-200

19. Ashburner J, Friston KJ. Voxel-based morphometry-the methods. NeuroImage 2000;11:805-21 
20. Good CD, Johnsrude IS, Ashburner J, et al. A voxel-based morphometric study of ageing in $\mathbf{4 6 5}$ normal adult human brains. NeuroImage 2001;14:21-36

21. Good CD, Johnsrude I, Ashburner J, et al. Cerebral asymmetry and the effects of sex and handedness on brain structure: a voxel-based morphometric analysis of 465 normal adult human brains. NeuroImage 2001;14:685-700

22. Finger S. The Origins of Neuroscience: A History of Explorations into Brain Function. New York: Oxford University Press; 1994

23. Bentivoglio M. Cortical structure and mental skills: Oskar Vogt and the legacy of Lenin's brain. Brain Res Bull 1998;47:291-96

24. Spitzka EA. A study of the brains of six eminent scientists belonging to the American Anthropometric Society: together with a description of the skull of Professor E. D. Cope Trans Am Philos Soc 1907;21:175-308

25. Meyer A. The search for a morphological substrate in the brains of eminent persons including musicians: a historical review. In: Critchley M, Henson RA. Music and the Brain. London: Heinemann; 1977:255-81

26. Witelson SF, Kigar DL, Harvey T. The exceptional brain of Albert Einstein. Lancet 1999;353:2149-53

27. May A, Hajak G, Gan $\beta$ bauer S, et al. Structural brain alterations following 5 days of intervention: dynamic aspects of neuroplasticity. Cereb Cortex 2007;17:205-10

28. Dehaene S, Spelke E, Pinel P, et al. Sources of mathematical thinking: behavioral and brain-imaging evidence. Science 1999;284:970-74

29. Cohen L, Dehaene S, Chochon F, et al. Language and calculation within the parietal lobe: a combined cognitive, anatomical and fMRI study. Neuropsychologia 2000;38:1426-40

30. Stanescu-Cosson R, Pinel P, van De Moortele PF, et al. Understanding dissociations in dyscalculia: a brain imaging study of the impact of number size on the cerebral networks for exact and approximate calculation. Brain 2000;123:2240-55

31. Hadamard J. The Psychology of Invention in the Mathematical Field. NJ: Princeton University Press; 1949:142-43

32. Black JE, Isaacs KR, Anderson BJ, et al. Learning causes synaptogenesis, whereas motor activity causes angiogenesis, in cerebellar cortex of adult rats. Proc Natl Acad Sci U S A 1990;87:5568-72

33. Isaacs KR, Anderson BJ, Alcantara AA, et al. Exercise and the brain: angiogen- esis in the adult rat cerebellum after vigorous physical activity and motor skil learning. J Cereb Blood Flow Metab 1992;12:110-19

34. Anderson BJ, Li X, Alcantara AA, et al. Glial hypertrophy is associated with synaptogenesis following motor-skill learning, but not with angiogenesis following exercise. Glia 1994;11:73-80

35. Kempermann G, Kuhn HG, Gage FH. More hippocampal neurons in adult mice living in an enriched environment. Nature 1997;386:493-95

36. Kempermann G, Kuhn HG, Gage FH. Experience-induced neurogenesis in the senescent dentate gyrus. J Neurosci 1998;18:3206-12

37. Butefisch CM, Davis BC, Wise SP, et al. Mechanisms of use dependent plasticity in the human motor cortex. Proc Natl Acad Sci U S A 2000;97:3661-65

38. Barinaga M. Developmental biology. Newborn neurons search for meaning. Science 2003;299:32-34

39. Ehninger D, Kempermann G. Regional effects of wheel running and environmental enrichment on cell genesis and microglia proliferation in the adult murine neocortex. Cereb Cortex 2003;13:845-51

40. van Turennout M, Bielamowicz L, Martin A. Modulation of neural activity during object naming: effects of time and practice. Cereb Cortex 2003;13:381-91

41. Kempermann G, Wiskott L, Gage FH. Functional significance of adult neurogenesis. Curr Opin Neurobiol 2004;14:186-91

42. Kleim JA, Hogg TM, VandenBerg PM, et al. Cortical synaptogenesis and motor map reorganization occur during late, but not early, phase of motor skill learning. J Neurosci 2004;24:628-33

43. Song H, Kempermann G, Overstreet Wadiche L, et al. New neurons in the adult mammalian brain: synaptogenesis and functional integration. J Neurosci 2005;25:10366-68

44. Barnea A, Nottebohm F. Seasonal recruitment of hippocampal neurons in adult free-ranging black-capped chickadees. Proc Natl Acad Sci USA 1994;91:11217-21

45. Kirn KJ, O'Loughlin B, Kasparian S, et al. Cell death and neuronal recruitment in the high vocal center of adult male canaries are temporally related to changes in song. Proc Natl Acad Sci U S A 1994;91:7844-48

46. Aydin K, Ciftci K, Terzibasioglu E, et al. Quantitative proton MR spectroscopic findings of cortical reorganization in the auditory cortex of musicians. AJNR Am J Neuroradiol 2005;26:128-36 